\title{
Testicular germ cells tumors in adolescents and young adults: Management and outcomes from a single-center experience
}

\author{
Claudio Spinelli ${ }^{1}$, Gianmartin Cito ${ }^{2}$, Girolamo Morelli ${ }^{3}$, Marco Ghionzoli ${ }^{1}$, Alessia Bertocchini ${ }^{1}$, \\ Beatrice Sanna ${ }^{1}$, Luca Galli ${ }^{4}$, Andrea Antonuzzo ${ }^{5}$, Riccardo Morganti ${ }^{6}$, Silvia Strambi ${ }^{1}$ \\ ${ }^{1}$ Division of Pediatric, Adolescents and Young Adults Surgery, Department of Surgical Pathology, Medical, Molecular \\ and Critical Area, University of Pisa, Pisa, Italy; \\ 2 Department of Urology and Andrology Surgery, Careggi Hospital, University of Florence, Florence, Italy; \\ ${ }^{3}$ Department of Urology and Andrology Surgery, University of Pisa, Pisa, Italy; \\ ${ }^{4}$ Medical Oncology II, University of Pisa, Department of Translational Research and New Technologies in Medicine and Surgery, \\ University of Pisa, Pisa, Italy; \\ ${ }^{5}$ Medical Oncology I, National Health Service Department of Translational Medicine Research and New Technologies in Medicine \\ and Surgery, University of Pisa, Pisa, Italy; \\ ${ }^{6}$ Section of Statistics, Department of Clinical and Experimental Medicine, University of Pisa, Pisa, Italy.
}

\begin{abstract}
Summary Objective: To investigate and compare the effectiveness of active surveillance versus post-surgical active treatment, in patients with testicular germ cells tumor (TGCT).

Materials and methods: We retrospectively analyzed 52 patients who underwent surgery for TGCT from January 2009 to December 2014. All the patients were divided into two age groups: the Group A included children-adolescents from 18 months to 21 years old, while the Group B comprised young adults from 22 to 39 years old. Clinical, histopathological, therapeutic and follow-up data were collected.

Results: Overall, 22 patients (42,3\%) were enrolled in the Group A and 30 patients (57.7\%) were categorized in the Group B. Inguinal orchiectomy was performed in all patients.

Retroperitoneal lymphadenectomy was performed in 4 patients (7.7\%). Post-surgical management differed based on clinical stage, resulting in active surveillance or adjuvant therapy. After an average 7 years follow-up period (range: 3.5-9.0 years), the overall survival rate is $100 \%$. The relapse risk is significantly higher for the patients in the Group B, displaying a recurrence free-survival rate of $72 \%$ versus $95 \%$ (Group A); 11 relapses (21.1\%) were recorded 2 years after surgery. Of these, 3 recurrences $(12.0 \%)$ occurred in patients undergoing an active surveillance approach, while 8 (29.6\%) in patients subjected to an active treatment.

Conclusions: The excellent prognosis in both age groups confirms the high curability of this neoplasia. The active surveillance could represent an optimal option for low recurrence risk tumors. However, post-surgical treatments should be taken into consideration for TGCT with high risk factors, including tumor size, lymphovascular and rete testis invasion.
\end{abstract}

KEY WORDS: Testicular germ cell tumors; Surgery; Children; Young adults; Active surveillance.

Submitted 10 March 2021; Accepted 7 May 2021

\section{INTRODUCTION}

Testicular germ cell tumor (TGCT) is a rare form of cancer in childhood, adolescence and young adulthood. Despite its infrequency (only $1 \%$ of male frequencies in the United States), TGCT represents the most common malignancy in young men between 20-39 years old in Northern and Southern Europe (the peak age of incidence is 30 years) (1).

The incidence of TGCT has been increasing in the developed countries for at least four decades (2-4). Rates vary by ethnic group: white men are at a higher risk for TGCT, with an annual incidence of $6.6 / 100,000$, compared to $1.4 / 100.000$ in black men and $1.9 / 100.000$ in Asians/Pacific Islanders (5, 6).

The pathogenesis of TGCT is multifactorial, including both genetic and environmental factors (7-9). A relationship between cryptorchidism and testicular cancer is well known (10). However, the risk factors for testicular cancer are not well characterized. A previous unilateral testicular cancer and a family history of testicular tumor are the only other factors clearly associated with increased risk. So far, studies have estimated an increased risk of TGCT 8-10 times fold and 4-6 times fold in brothers and sons respectively (7).

Nonetheless, mortality rates have dropped significantly over the past 3 decades owing to the development of more effective treatments (2). The aim of this study was to investigate the clinical outcomes of TGCT in two different age groups treated with active surveillance (AS) or active treatment (AT), according to the histopathological findings and the stage of disease.

\section{MATERIALS AND METHOdS}

We retrospectively analyzed 52 patients who underwent surgery for TGCT from January 2009 to December 2014. All the patients were divided into two age groups: the Group A included children-adolescents from 18 months to 21 years old, while the Group B comprised young adults from 22 to 39 years old. Clinical, histopathological, therapeutic and follow-up data were collected. For

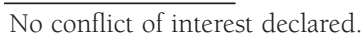


the guidance during diagnostic assessment, tumor markers levels were recorded, including human chorionic gonadotropin (HCG), alpha-fetoprotein (AFP) and lactate dehydrogenase (LDH). Total testosterone, estradiol, follicle-stimulating hormone (FSH) and luteinizing hormone (LH) levels were collected pre-operatively. In order to define the pre-surgical tumor stage, all patients underwent thorax and abdomen imaging scans, including contrast-enhancement computed tomography (CT) or magnetic resonance (MR). We selected the treatments for patients according to the European Association of Urology (EAU) guidelines. AS is considered a feasible approach in clinical stage (CS) I seminoma testis patients. The AS protocol after surgery has involved the evaluation of tumor marker levels every 2 months in the first two years, every 4 months during the third year, every 6 months during the fourth year, and then yearly from the fifth year onwards. Chest radiography or abdominal/pelvic CT/MRI, color doppler ultrasonography (US) of testicles, abdomen and pelvis were performed every 6 months for the first 3 years and then, abdominal CT/MRI after the fifth year.

All the patients gave the oral and written consent on management options. Moreover, they have been fully informed about the risk of recurrence during the active surveillance approach. For patients with higher cancer stage at diagnosis, active treatment was preferred.

Meanwhile, disease relapse was defined as imaging or physical examination evidence of metastases and/or elevated tumor markers.

Follow-up data of the AS protocol or the post-surgical AT approach were collected. Moreover, the overall survival (OS) rate and recurrence free survival (RFS) rate was measured.

\section{Data analysis}

The authors confirm the availability of, and access to, all original data reported in this study. Categorical data were described by absolute and relative frequency. In order to compare the histologic categories (seminomas, non-seminomas) in the Group A and the Group B in different stages (IS, I, II, III), the z-test for two proportions was applied. An RFS analysis was performed using the Kaplan-Meier method and the log-rank test was used to detect differences between "Group A" and "Group B" curves. The significance was fixed at $<0.05$. All analyses, descriptive and inferential, were carried out by SPSS v.26.

\section{RESULTS}

The Group A (children-adolescents) included 22 patients (42.3\%) and the Group B (young adults) comprised 30 patients (57.7\%). The median age in Group A was 16.0 years (range: 18.0 months-21.0 years), while the median age in Group B was 28.0 years (range: 22.0-39.0 years). In the Groups B, 2/30 patients (6.6\%) reported a paternal family history of seminoma, and $1 / 30$ patient (3.3\%) underwent surgery for cryptorchidism.

The most common clinical presentation was a palpable and painless testicular mass in $42 / 52$ cases $(80.7 \%$ ), or testis swelling in $10 / 52$ patients (19.2\%).

Three patients (10.0\%) in the Group B showed distant metastasis at diagnosis: 1 patient $(3.3 \%)$ had cervical supraclavicular lymph node metastasis as primary clinical presentation and 2 patients $(6.7 \%)$ had mediastinal lymph node metastasis associated with respiratory symptoms (wheezing, coughing and chest tightness).

All patients reported total testosterone, estradiol, FSH and $\mathrm{LH}$ levels, in the normal range. Tumor markers were expressed in $14 / 52$ cases (26.9\%). In all patients with increased tumor markers, normal levels were reached within 12 months after surgery. The median US tumor size was $20.0 \mathrm{~mm}$ in the Group A (range: $10.0-70.0 \mathrm{~mm}$ size) and $22.4 \mathrm{~mm}$ in the Group B (range: 10.0-50.0 mm). Inguinal orchiectomy was performed in all cases. One patient in the Group A underwent a scrotal incision due to high volume tumor mass. RPLND was performed simultaneously with orchiectomy in 4 patients $(7.7 \%)$, resulting in lymph-node involvement at the final pathological analysis. Frozen section examination was performed in 33 patients (63.5\%): 14 cases in the Group A (63.5\%) and 19 cases in the Group B (36.5\%). A testicular prosthesis was placed in 44 patients $(84.6 \%)$ : 17 in the Group A (77.3\%) and 27 in the Group B (90\%).

The prosthesis insertion occurred during orchiectomy in 42 cases (95.5\%) and after surgery in 2 cases (4.5\%).

Histopathological examination was carried out.

In 26 cases (50.0\%) typical Seminomatous Germ Cell Tumor (SGCT) was reported: 7 (31.8\%) in the Group A and $19(63.3 \%)$ in the Group B. Twenty-six (50.0\%) examinations detected typical Non Seminomatous Germ Cell Tumor (NSGCT): 15 (68.2\%) in the Group A and 11 (33.7\%) in the Group B. The histologic distribution of TGCT is reported in Table 1.

Our study showed that NSGCT are significantly more frequent $(\mathrm{p}$-value $=0.050)$ in children-adolescents, rather than in adults. A histopathology comparison between the two

Table 1.

Histologic distribution of TGCT. Statistics: frequency (\%).

\begin{tabular}{|l|c|c|c|c|}
\hline Histology & Total & Group A ( $\mathbf{n}=\mathbf{2 2})$ & Group $\mathbf{B}(\mathbf{n}=\mathbf{3 0})$ & p-value \\
\hline SGCT & $26(50)$ & $7(31.8)$ & $19(63.3)$ & 0.050 \\
\hline NSGCT & $26(50)$ & $15(68.2)$ & $11(33.7)$ & - \\
\hline Embryonal carcinoma & $19(36.5)$ & $11(50)$ & $8(26.6)$ & 0.150 \\
\hline Yolk sac tumor & $4(7.7)$ & $4(18.2)$ & $0(0)$ & 0.057 \\
\hline Mixed & $3(5.7)$ & $0(0)$ & $3(10)$ & 0.354 \\
\hline
\end{tabular}

Table 2.

Histologic category compared between Group A and Group B in different stages. Statistics: frequency (\%).

\begin{tabular}{|ll|c|c|c|}
\hline Stage & & Group A & Group B & p-value \\
\hline Stage IS & SGCT & $1(4.6)$ & $1(3.3)$ & 0.625 \\
& NSGT & $0(0)$ & $0(0)$ & - \\
\hline Stage I (a/b) & SGCT & $5(22.8)$ & $11(36.7)$ & 0.442 \\
& NSGT & $13(59)$ & $5(16.7)$ & 0.04 \\
\hline Stage II (a/b/c) & SGCT & $1(4.6)$ & $6(20)$ & 0.232 \\
& NSGT & $2(9)$ & $5(16.7)$ & 0.630 \\
\hline Stage III (a) & SGCT & $0(0)$ & $1(3.3)$ & 0.868 \\
& NGCT & $0(0)$ & $1(3.3)$ & 0.868 \\
\hline Total & & 22 & 30 & \\
\hline
\end{tabular}




\begin{tabular}{|l|c|ccccc|ccccc|}
\hline & & \multicolumn{5}{|c|}{ Group A (22 cases) } & \multicolumn{5}{c|}{ Group B (30 cases) } \\
\hline Histology & Stage & AS & RT & CT & CT+RT & RPLND & AS & RT & CT & CT+RT & RPLND \\
\hline SGCT & IS & $1(4.5)$ & - & - & - & - & $1(3.3)$ & - & - & - & - \\
& I & $5(22.7)$ & - & - & - & - & $7(23.3)$ & $1(3.3)$ & - & $2(6.7)$ & $1(3.3)$ \\
& II & - & - & $1(4.5)$ & - & - & - & - & $5(16.7)$ & $1(3.3)$ & - \\
& III & - & - & - & - & - & - & - & - & $1(3.3)$ & - \\
\hline NSGT & IS & - & - & - & - & - & - & - & - & - & - \\
& I & $13(59.1)$ & - & $2(9.1)$ & - & - & $5(16.7)$ & - & - & - & - \\
& II & - & - & - & - & - & - & - & $2(6.7)$ & - & $3(10)$ \\
& III & - & - & - & - & - & - & - & $1(3.3)$ & - & - \\
& & & & & - & & & & & \\
\end{tabular}

Table 3.

Post-surgical management of SGCT-NSCT, comparison between Group A and Group $B$ (AS: Active Surveillance; RT: Radiotherapy; CT: Chemotherapy; RPLND: Retroperitoneal Lymphadenectomy). Statistics: frequency (\%).

groups in different stages is reported in Table 2 . Comparison of tumor stages at presentation did not show significant differences among groups. AS was performed in 32 patients (61.5\%), while AT was performed in 20 patients (38.5\%). Post-surgical management is reported in Table 3. After an average follow-up period of 7.0 years (range: 3.5 9.0 years), 11 relapses (21.1\%) were recorded within the first 2 years after orchiectomy.

Recurrence was higher in Group B although we must highlight that initial stage distribution was not homogeneous in the two groups. Recurrences occurred respectively in retroperitoneal lymphnodes, in retroperitoneal and retromediastinal lymph nodes, or in retroperitoneal lymph nodes with lung metastasis. Each of these cases presented with increased tumor markers. Out of them, 1 patient underwent RPLND, while the other ones underwent systemic chemotherapy.

Overall, 8 recurrences (25.0\%) were recorded in the AT sample, of which 1 case (5.0\%) belonging to the Group A was diagnosed as NSGCT, involved the retroperitoneal lymph nodes and was treated with RPLND.

The Group B had 7 cases (35.0\%) of recurrence, all of them treated with chemotherapy and radiotherapy: 5 of them were SGCT and 2 cases were NSGCT. All those 7 patients showed a tumor mass greater than $3 \mathrm{~cm}$, local lymphovascular invasion (LVI) signs and infiltration of the rete testis. The overall survival rate is $100 \%$. Furthermore, as shown in Figure 1, the relapse risk is significantly higher for the patients in the Group B, displaying a recurrence free-survival rate of $72 \%$ versus $95 \%$ (Group A).

Figure 1.

Recurrence Free-survival analysis between Group A (rate 95\%) and Group B (rate 72\%).

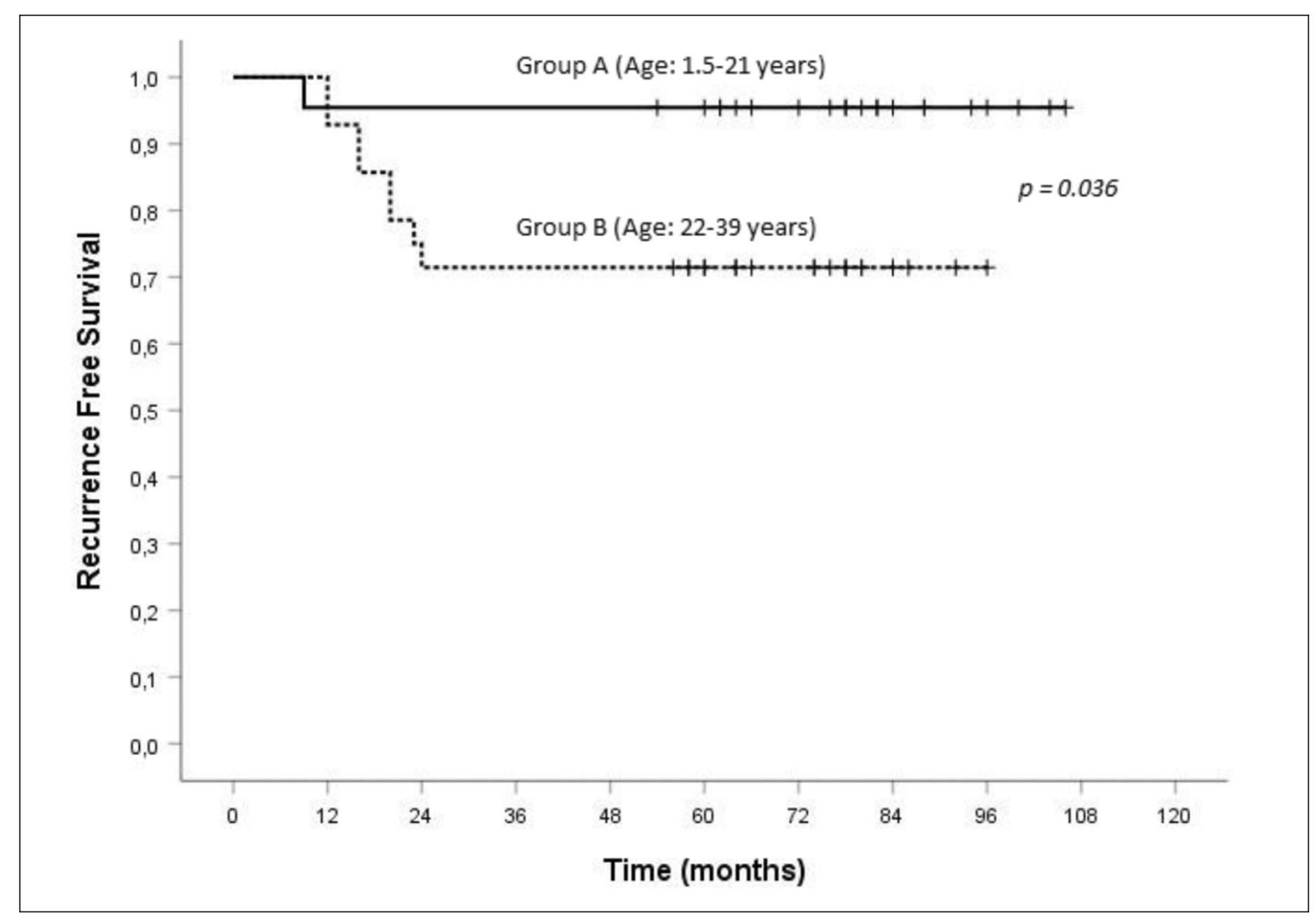




\section{Discussion}

Testicular cancer is largely found in young and middle-age men, but around 7\% of cases occur in children (11-19). TGCTs represent $71 \%$ of all testicular neoplasms and they include yolk sac tumors, teratoma, seminoma, choriocarcinoma and embryonal carcinoma (20). Gonadal stromal tumors (NTGCTs) include Leydig cell tumor, Sertoli cell tumor, juvenile granulosa cell tumor and gonadoblastoma $(21,22)$. As reported in literature, testicular tumors may be different, based on age-related range in histopathology, molecular biology, malignant potential, clinical behavior and treatment $(12,22-24)$. Moreover, malignant potential is significantly lower in the pediatric age group compared to the other age groups (25).

In our study, the incidence of NSGCT is higher in the children's group than in the young-adults group ( $\mathrm{p}=$ 0.04). An important role in the diagnosis and follow-up is played by serum tumor markers $(23,26)$. In our case series, tumor markers were expressed in $26,9 \%$ of cases. This was true for both age groups for NSGCT, where markers reflect tumor widespread, aggressiveness and constitute a prognostic factor for the cancer itself (18); in the young adults group, markers were expressed only in SGCT. After surgical treatment, we reached normal levels of serum tumor markers in absence of metastatic disease. On the other hand, we found tumor markers increasing in patients with relapse, in accordance with data reported the literature (24-26).

Treatment of the primary TGCT is performed by radical orchiectomy. Post-surgical treatment was based on histopathologic features and disease stage at surgery (2, $27,28)$. Management options after surgery included surveillance, adjuvant chemotherapy, radiotherapy and RPLND $(2,30)$. Considering the relatively low risk of relapse in testicular cancer, many guidelines recommend surveillance as the preferred initial treatment for all stage I SGCT and low-risk stage I NSGCT (31). Therefore, active surveillance has also been recently adopted by some cancer centers for high-risk stage NSGCT, considering that many patients do not require further therapy and those with relapses can be treated with highly effective salvage therapy $(16,27)$.

Regarding SGCT, the recent strategic algorithm considers surveillance alone for stage I patients with almost 100\% of overall survival. Eventual relapses may be cured by radiation or chemotherapy (2). Adjuvant treatments have declined in recent decades, as surveillance has been increasingly used to avoid unnecessary treatment and related long-term toxicities $(2,30)$. Indeed, historically active surveillance became an option in the 1980's when it was demonstrated that cisplatin-based chemotherapy could cure almost all recurrences. Today, it is the management option suggested by the guidelines because it has nearly the same overall survival rate of other adjuvant treatments as well as being a safe and non-invasive option in selected cases (3, 30-32).

The trend nowadays is a de-escalation of therapy toward AS for early stage testicular cancers.

The main debate against adjuvant chemotherapy is due to the lack of improved overall survival and the association with long-term side effects, including infertility, secondary malignancies, increased risk for cardiovascular disease, impaired kidney function, hearing impairment and peripheral neuropathy $(2,30,33,34)$. Nappi et al. (34) found that active surveillance is highly relevant in avoiding overtreatment in $50-85 \%$ of patients, with no long-term side effects in non-relapsing patients and an overall survival of almost $100 \%$ even in patients with recurrent disease.

A study by Nayan et al. (35) conducted on 1239 patients with TGCT, treated with active surveillance after orchiectomy for clinical stadium I, showed that the risk percentage of relapse in the first 5 years after orchiectomy was $42.4 \%$ for high risk NSGCT, $17.3 \%$ for low risk NSGCT, $20.3 \%$ for SGCT more than $3 \mathrm{~cm}$ wide, and $12.2 \%$ for SGCT less than $3 \mathrm{~cm}$ wide.

In a study by Albers et al. (16) in patients with stage I NSGCT, the tumors had a 30\% risk of progression which required treatment.

Furthermore, it is known that the risk of relapse in Stage I NSGCT is substantially related to the presence of Lympho-Vascular Invasion (LVI), which implies a 30-50\% recurrence risk $(25,36)$.

Moreover, as discussed by Yilmaz et al. (37) in their study, LVI could be considered a prognosticating indicator for NSGCT, thus the status of rete testis and testicular hilum should be taken into consideration when choosing therapies.

Cohn-Cedermark et al. (38) advice chemotherapy for patients with at least two risk factors like tumor size and invasion of the rete testis. High-risk patients can also be managed with initial surveillance to spare the $50 \%$ in whom disease will not progress, but other studies recommend precautionary chemotherapy (33-35). Recurrences occur most commonly in the retroperitoneum, with the majority diagnosed within 2 years after orchiectomy (39, 40). Although recurrence rates are not comparable in the two groups due to different initial staging, in our study we observed relapses in the first 2 years after orchiectomy preeminently in the retroperitoneum, in $32.2 \%$ of the patients who underwent AT compared to $11.1 \%$ of patients submitted to AS. In the patients with AT, LVI and rete testis signs of infiltration were reported (45.5\% NSGCT and $27.3 \%$ SGCT).

All the relapses in patients with AS were histological samples of NSGCT in the Group B. All the recurrences in both groups presented a tumor mass greater than $3 \mathrm{~cm}$ in size.

\section{Conclusions}

AS proves to be a feasible option for stage I TGCT, whereas post-surgical therapy requires to be performed for higher stage of TGCT.

The Kaplan-Meier curve shows a significant difference of RFS for younger patients although they presented with different stage at presentation. Considering the overall excellent outcomes of AS both in terms of OS and RFS, our experience suggests that post-orchiectomy active treatments might be limited to selected patients with well-known relapse risk factors, such as tumor size, lymphovascular and rete testis invasion, while the other patients could benefit from an accurate active surveillance approach. 


\section{ACKNOWLEDGMENTS}

A special thanks to Helen Romito from Sant'Anna School of Advanced Studies in Pisa for her editing work.

\section{REFERENCES}

1. Hayes-Lattin B, Bleyer A. "Testicular Cancer" in Cancer in adolescents and young adults. Springer Publishing AG, 2017.

2. Ehrlich Y, Margel D, Lubin MA, et al. Advances in the treatment of testicular cancer. Transl Androl Urol. 2015; 4:381-90.

3. Guzzinati S, Buzzoni C, De Amgelis R, et al. ARTUM working group. Cancer prevalence in Italy: an analysis of geographic variability. Cancer Causes Control, 2012; 23:1497-510.

4. Shen AH, Howell D, Edwards E, et al. The experience of patients with early-stage testicular cancer during the transition from active treatment to follow-up surveillance. Urol Oncol. 2016; 34:168.e11-20.

5. McGlynn KA, Trabert B. Adolescent and adult risk factors for testicular cancer. Nature Reviews Urology. 2012; 9:339-49.

6. Chien FL, Schwartz SM, Johnson RH. Increase in testicular germ cell tumor incidence among hispanic adolescents and young adults in the United States. Cancer 2014; 120:2728-34.

7. Schmiedel S, Schuz J, Skakkebaek NE, et al. Testicular germ cell cancer incidence in an immigration perspective, Denmark, 1978 to 2003. The J Urol. 2010; 183:1378.

8. Erin L. Marcotte, Pankratz N, Amatruda J Fetal. Variants in BAK1, SPRY4 and GAB2 are Associated with pediatric germ cell tumors: are port from the children's oncology group. Genes Chromosomes Cancer. 2017; 56:548-558.

9. Le Cornet C, Fervers B, Pukkala E, et al. Parental occupational exposure to organic solvents and testicular germ cell tumors in their offspring: NORD-TEST study. Environ Health Perspect. 2017; 125:067023.

10. Turnbull C, Rapley EA, Seal S, et al. Variants near DMRT1, TERT and ATF7IP are associated with testicular germ cell cancer. Nat Genet. 2010; 42:604-7.

11. DeRouen MC, Mujahid M, Srinivas S, et al. Disparities in adolescent and young adult survival after testicular cancer vary by histologic subtype: a population-based study in California 1988-2010. J Adolesc Young Adult Oncol. 2016; 5:31-40.

12. Leslie SW, Villanueva CA. Cryptorchidism. In Stat Pearls, Treasure Island (FL)-Stat Pearls Publishing 2018.

13. Shaw PH, Reed DR, Yeager N, et al. Adolescent and young adult (AYA) oncology in the United States: a specialty in its late adolescence. J Pediatr Hematol Oncol. 2015; 37:161-9.

14. Hisamatsu E, Takagi S, Nakagawa Y, et al. Prepubertal testicular tumors: A 20-year experience with 40 cases. Int J Urol. 2010; 17:956-9.

15. Sobin LH, Gospodarowicz MK, Wittekind Ch. TNM Classification of Malignant Tumors, International Union Against Cancer. 2009.

16. Albers P, Albrecht W, Algaba F, et al. European Association of Urology. Guidelines on Testicular Cancer: 2015 Update. Eur Urol. 2015; 68:1054-68.

17. Oldenburg J, Fosså SD, Nuver J, et al. ESMO Guidelines Working Group. Testicular seminoma and non-seminoma: ESMO Clinical Practice Guidelines for diagnosis, treatment and follow-up. Ann Oncol. 2013; 24 Suppl 6:vi125-32.

18. Tryakin A, Fedyanin M, Bulanov A, et al. Dose-reduced first cycle of chemotherapy for prevention of life-threatening acute com- plications in nonseminomatous germ cell tumor patients with ultra high tumor markers and/or poor performance status. J Cancer Res Clin Oncol. 2018; 144:1817-1823.

19. EAU Guidelines. Edn. presented at the EAU Annual Congress Milan 2021. ISBN 978-94-92671-13-4.

20. Boccellino M, Vanacore D, Zappavigna S, et al. Testicular cancer from diagnosis to epigenetic factors. Oncotarget, 2017; 8:104654104663.

21. Abhomela M. Pediatric Testicular Cancer: two decades of Saudi national data. Urol Ann. 2017; 9:310-314.

22. Chung JM, Lee SD. Overview of pediatric testicular tumors in Korea. Korean Journal of Urology. 2014; 55:789-96.

23. Sun H, Kim P, Jia P, et al. Distinct telomere length and molecular signatures in seminoma and non-seminoma of testicular germ cell tumor. Brief Bioinform. 2019; 20:1502-1512.

24. Nistal M, Paniagua R, González-Peramato P, et al. Perspective in pediatric pathology, Chapter 25. Testicular and paratesticular tumors in the pediatric age group. Pediatr Dev Pathol. 2016; 19:471-492.

25. Hoffman HA, Toshkezi G, Fullmer JM, et al. Pitfalls in diagnosis and management of testicular choriocarcinoma metastatic to the brain: report of 2 cases and review of literature. World Neurosurgery. 2017; 106:536-542.

26. Murray MJ, Huddart RA, Coleman N. The present and future of serum diagnostic tests for testicular germ cell tumors. Nat Rev Urol. 2016; 13:715-725.

27. Romo Muñoz MI, Núñez Cerezo V, Dore Reyes $M$, et al. Tumores testiculares en la edad pediátrica: indicaciones de la cirugía conservadora [Testicular tumours in children: Indications for testissparing surgery]. An Pediatr (Engl Ed). 2018; 88:253-258.

28. Kamran SC, Seisen T, Markt SC, et al. Contemporary treatment patterns and outcomes for clinical stage IS testicular cancer. Eur Urol. 2018; 73:262-270.

29. Ehrlich Y, Beck SD, Foster RS, et al. Serum tumor markers in testicular cancer. Urol Oncol. 2013; 31:17-23.

30. Gumus M, Bilici A, Odabas H, et al. Outcomes of surveillance versus adjuvant chemotherapy for patients with stage IA and IB non seminomatous testicular germ cell tumors. World J Urol. 2017; 35:1103-1110.

31. Alanee S, Nicholson A, Slaton J. Contemporary treatment of low risk stage I non-seminomatous germ cell testicular tumors: a survey of the society of Urologic Oncology. Urol. Oncol. 2012; 30:749-51.

32. Patel HD, Srivastava A, Alam R, et al. Radiotherapy for stage I and II testicular seminomas: secondary malignancies and servival. Urol Oncol. 2017; 35:606.e1- e7.

33. Leveridge MJ, Siemens DR, Brennan K, et al. Temporal trends in management and outcomes of testicular cancer: A population-based study. Cancer. 2018; 124:2724-2732.

34. Nappi L, Nichols CR, Kollmannsberger CK. New treatments for stage I testicular cancer. Clinical Adv Hematol Oncol. 2017; 15:626631.

35. Nayan M, Jewett MAS, Hosni A, et al. Conditional risk of relapse in surveillance for clinical stage I testicular cancer. Eur Urol. 2017; 71:120-127.

36. Richie JP. Cardiovascular Disease Mortality after chemotherapy or surgery for testicular non seminoma:a population-based study. J Urol. 2016; 196:1448-1449.

37. Yilmaz A, Cheng T, Zhang J, Trpkov K. Testicular hilum and 
vascular invasion predict advanced clinical stage in nonseminomatous germ cell tumors. Mod Pathol. 2013; 26:579-86.

38. Cohn-Cedermark G, Stahl O, Tandstad T. SWENOTECA. Surveillance vs.adjuvant therapyof clinical stage I testicular tumors-a review and the SWENOTECA experience. Andrology. 2015; 3:102-10.
39. Smart, Lopes, Rice S, et al. Chemotherapy drugs cyclophosphamide, cisplatin and Doxorubicin induce germ cell loss in an in vitro model of the prepubertal testis. Sci Rep. 2018; 8:1773.

40. Chung P, Warde P. Contemporary management of stage I and II seminoma. Curr Urol Rep. 2013; 14:525-33.

\section{Correspondence}

Claudio Spinelli, MD

claudio.spinelli@unipi.it

Marco Ghionzoli, MD

marcoghionzoli@hotmail.com

Alessia Bertocchini, MD

villinofibbiani@hotmail.com

Beatrice Sanna, MD

beatricesanna.md@gmail.com

Silvia Strambi, MD

sil.strambi@gmail.com

Division of Pediatric, Adolescents and Young Adults Surgery, Department of Surgical

Pathology, Medical, Molecular and Critical Area, University of Pisa, Pisa (Italy)

Gianmartin Cito, MD (Corresponding Author)

gianmartin.cito@gmail.com

Girolamo Morelli, MD

girolamomorelli@gmail.com

Department of Urology and Andrology Surgery, Careggi Hospital, University of Florence

Largo Brambilla, 350134 Florence (Italy)

Luca Galli, MD

lgalli@unipi.it

Medical Oncology II, University of Pisa, Department of Translational Research and New Technologies in Medicine and Surgery, University of Pisa, Pisa (Italy)

Andrea Antonuzzo, MD

aantonuzzo@unipi.it

Medical Oncology I, National Health Service Department of Translational Medicine

Research and New Technologies in Medicine and Surgery, University of Pisa, Pisa (Italy)

Riccardo Morganti, MD

r.morganti@ao-pisa.toscana.it

Section of Statistics, Department of Clinical and Experimental Medicine,

University of Pisa, Pisa (Italy) 